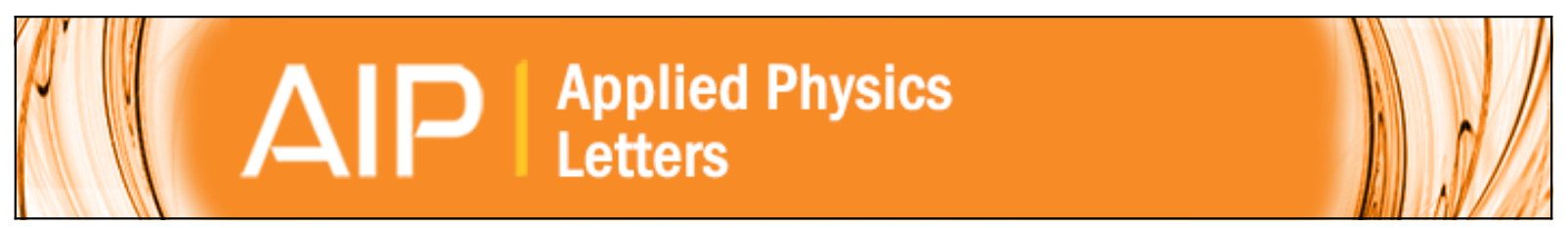

Polariton condensation in a strain-compensated planar microcavity with InGaAs quantum wells

Pasquale Cilibrizzi, Alexis Askitopoulos, Matteo Silva, Faebian Bastiman, Edmund Clarke, Joanna M. Zajac, Wolfgang Langbein, and Pavlos G. Lagoudakis

Citation: Applied Physics Letters 105, 191118 (2014); doi: 10.1063/1.4901814

View online: http://dx.doi.org/10.1063/1.4901814

View Table of Contents: http://scitation.aip.org/content/aip/journal/apl/105/19?ver=pdfcov

Published by the AIP Publishing

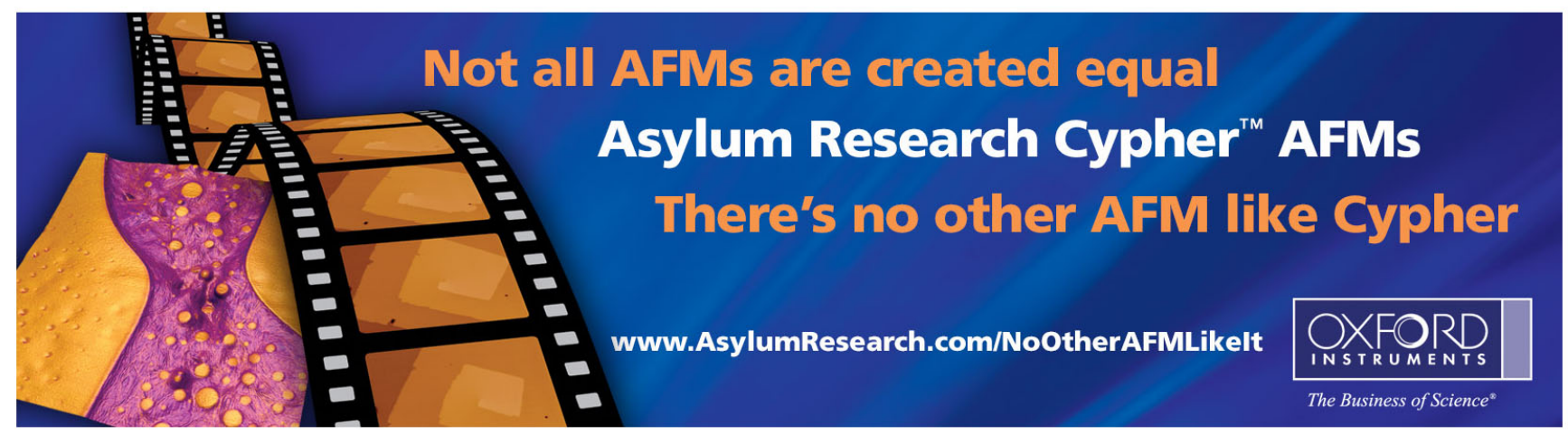




\title{
Polariton condensation in a strain-compensated planar microcavity with InGaAs quantum wells
}

\author{
Pasquale Cilibrizzi, ${ }^{1}$ Alexis Askitopoulos, ${ }^{1, a)}$ Matteo Silva, ${ }^{1}$ Faebian Bastiman, ${ }^{2}$ \\ Edmund Clarke, ${ }^{2}$ Joanna M. Zajac, ${ }^{3, b)}$ Wolfgang Langbein, ${ }^{3}$ and Pavlos G. Lagoudakis ${ }^{1}$ \\ ${ }^{1}$ Department of Physics and Astronomy, University of Southampton, Southampton SO17 1BJ, United Kingdom \\ ${ }^{2}$ EPSRC National Centre for III-V Technologies, University of Sheffield, Mappin Street, Sheffield S1 3JD, \\ United Kingdom \\ ${ }^{3}$ School of Physics and Astronomy, Cardiff University, The Parade, Cardiff CF24 3AA, United Kingdom
}

(Received 22 July 2014; accepted 23 October 2014; published online 14 November 2014)

\begin{abstract}
The investigation of intrinsic interactions in polariton condensates is currently limited by the photonic disorder of semiconductor microcavity structures. Here, we use a strain compensated planar GaAs/AlAs $0.98 \mathrm{P}_{0.02}$ microcavity with embedded InGaAs quantum wells having a reduced cross-hatch disorder to overcome this issue. Using real and reciprocal space spectroscopic imaging under non-resonant optical excitation, we observe polariton condensation and a second threshold marking the onset of photon lasing, i.e., the transition from the strong to the weak-coupling regime. Condensation in a structure with suppressed photonic disorder is a necessary step towards the implementation of periodic lattices of interacting condensates, providing a platform for on chip quantum simulations. (C) 2014 AIP Publishing LLC. [http://dx.doi.org/10.1063/1.4901814]
\end{abstract}

Planar exciton-polaritons are bosonic light-matter quasiparticles formed by the strong coupling between quantum well (QW) excitons and the photonic mode of a planar semiconductor microcavity (MC). ${ }^{1}$ Increasing the polariton population, stimulated scattering leads to a build up of polaritons in the ground state of the dispersion and gives rise to an inversion-less amplification of the polariton emission. ${ }^{2}$ The resulting macroscopic ground state population is a nonequilibrium Bose-Einstein condensate (BEC). ${ }^{3}$ Solid state polariton condensates have been used to explore fundamental concepts such as superfluidity and quantum vortices in polariton fluidics. ${ }^{4,5}$ The BEC phase transition has been demonstrated in a wide range of materials. Condensation at room temperature under optical excitation has been reported in $\mathrm{GaN}^{6}$ and more recently in $\mathrm{ZnO}^{7}$ and organic systems. ${ }^{8,9}$ In III-V materials, polariton condensation and lasing have been reported in MCs with GaAs QWs. ${ }^{10}$ The implementation of electrically injected polariton condensates has also been reported in $\mathrm{GaAs}^{11,12}$ and recently in $\mathrm{GaN}$ at room temperature. ${ }^{13}$ Among these materials, AlAs/GaAs MCs are currently the preferred system for the study of polariton fluidics due to low photonic and QW disorder combined with a moderate exciton binding energy $(\sim 10 \mathrm{meV})$. Nevertheless, the presence of cross-hatched dislocations affects the dynamics of polaritons giving rise to localization ${ }^{14}$ and scattering ${ }^{15-18}$ phenomena that limit the propagation of polaritons within the cavity plane. In our previous work, ${ }^{19}$ we have shown that one can reduce the cross-hatched dislocation density by introducing strain-compensating AlP layers into the centre of the AlAs layer of the distributed Bragg reflectors (DBRs). Here, we report on polariton condensation in a planar, strain compensated $2 \lambda$ GaAs microcavity with embedded InGaAs QWs under non-resonant optical

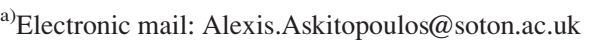

${ }^{b}$ Present address: School of Engineering and Physical Sciences, Heriot-Watt University, Edinburgh EH14 4AS, United Kingdom.
}

excitation. Strain compensation was achieved by $\mathrm{AlAs}_{0.98} \mathrm{P}_{0.02} / \mathrm{GaAs}$ DBR layers instead of the thin AlP inserts in the AlAs layers used in Ref. 19 as their effective composition could be better controlled. The bottom DBR consists of 26 pairs of GaAs and $\mathrm{AlAs}_{0.98} \mathrm{P}_{0.02}$, while the top has 23 of these pairs as shown in Fig. 1(a), resulting in very high reflectance $(>99.9 \%)$ in the stop-band region of the spectrum as shown in Fig. 1(b). Three pairs of $6 \mathrm{~nm}$ $\mathrm{In}_{0.08} \mathrm{Ga}_{0.92} \mathrm{As} \mathrm{QWs}$ are embedded in the GaAs cavity at the anti-nodes of the field as well as two additional QWs at the first and last nodes to serve as carrier collection wells, as shown in Fig. 1(c). The large number of QWs was chosen to increase the Rabi splitting and keep the exciton density per QW below the Mott density ${ }^{20}$ also for sufficiently high polariton densities to achieve polariton condensation. A wedge in the cavity thickness allows access to a wide range of exciton-cavity detuning. The average density of hatches along the [110] direction was estimated from transmission imaging to be about $6 / \mathrm{mm}$, while no hatches along the $[1 \overline{1} 0]$ direction were observed, as exemplified in Fig. 1(d).

In the experiments, the sample was held in a cold finger cryostat at a temperature of $T=6 \mathrm{~K}$. The optical excitation, for all the measurements reported in this work, was at the first reflectivity minimum above the cavity stop band, as shown in Fig. 1(b). The photoluminescence from the lower polariton (LP) was acquired with $\pm 40^{\circ}$ collection angle, by a 0.65 NA microscope objective and was spectrally resolved using a 1200 grooves $/ \mathrm{mm}$ grating in a $300 \mathrm{~mm}$ spectrometer coupled to a cooled charge coupled device. The spectrally and in-plane wavevector $(k)$ resolved emission intensity at low excitation fluence and at a detuning of $\Delta=-5 \mathrm{meV}$ is shown in Fig. 1(e). Extracting the upper polariton (UP) and LP energy at $k \sim 0$ across the thickness gradient of our sample, we find the detuning dependence shown in Fig. 1(f). To fit the experimental data, we use a system of two coupled harmonic oscillators. ${ }^{21}$ The strong coupling between the exciton resonance and the cavity mode is observed with a 

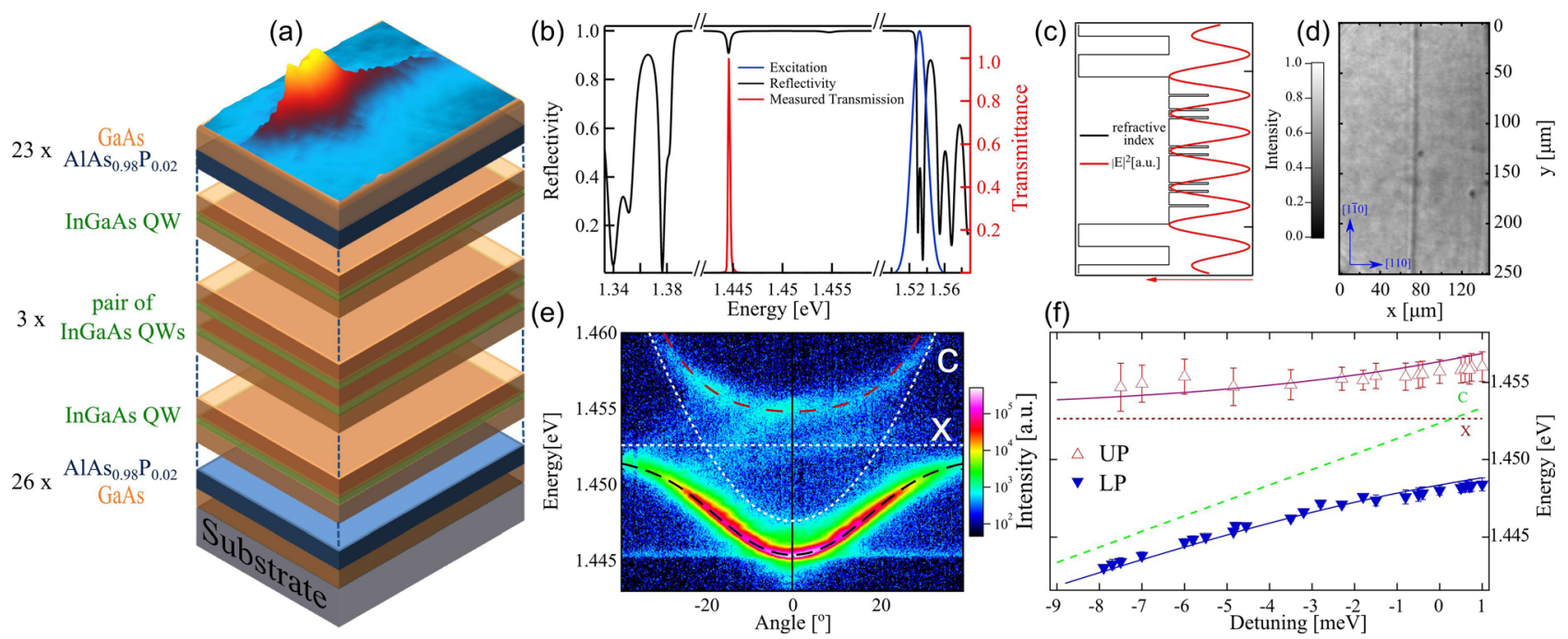

FIG. 1. (a) Sketch of the microcavity structure and condensate emission. (b) Calculated reflectivity of the cavity stop band with the transfer matrix method (black line), spectra of pulsed excitation (blue), and experimental transmittance spectrum (red) for detuning $\Delta=-5.8 \mathrm{meV}$. (c) Sketch of the refractive index (black line) along the growth direction and the corresponding square of the electric field of the cavity mode (red line). (d) Real space transmission intensity image of the sample surface under white light illumination on a linear gray scale, as indicated. (e) Polariton dispersion at low excitation fluence on a logarithmic color scale as given. The white dashed lines depict the bare exciton (X) and cavity (C) modes and the blue and red solid lines are the calculated UP and LP dispersions. (f) UP and LP energy at normal incidence for different detuning conditions. The error bars correspond to the FWHM of a Gaussian fit to the spectra, the blue (purple) line show the calculated UP (LP), and the dashed green (red) line shows the bare cavity (exciton) mode.

vacuum Rabi-splitting of $2 \hbar \Omega \sim 8 \mathrm{meV}$. From the transmitted spectra at $\Delta=-5.8 \mathrm{meV}$ shown in Fig. 1(b), at which the LP has a (resolution corrected) linewidth of $120 \pm 50 \mu \mathrm{eV}$ and an exciton fraction of $20.5 \%$, we obtain a LP Q-factor of $\sim 12000$ while the calculated bare cavity Q-factor, neglecting in-plane disorder and residual absorption, is $\sim 25000{ }^{22}$ As the emission energy of the InGaAs QWs is lower than the absorption of the GaAs substrate, we can study the photoluminescence of the sample both in reflection and transmission geometry. The transmission geometry, which is not available for GaAs QWs, allows to filter the surface reflection of the excitation and has been widely utilized to probe the features of polariton fluids ${ }^{23,24}$ under resonant excitation of the polaritons. We use non-resonant excitation from the epi side and detect the emission from the substrate side, so that the excitation is filtered by the absorption of the GaAs substrate.

In order to achieve condensation into the LP ground state at $k \sim 0$, we excite with a spot of $35 \mu$ m full width half maximum (FWHM). The optical excitation pulses of $180 \mathrm{fs}$ duration and $80 \mathrm{MHz}$ repetition rate are provided by a Ti:sapphire laser. They excite electron-hole pairs in the InGaAs QWs and GaAs cavity which rapidly relax to populate the LP dispersion and the weakly coupled QW exciton reservoir. With increasing exciton and polariton density, the polariton relaxation rate increases, eventually overcoming the threshold for condensation when the relaxation into the ground state of the LP supersedes its radiative decay, resulting in a macroscopic ground state population. ${ }^{3}$ Fig. 2(a) shows the energy and wave-vector resolved emission intensity in the low fluence regime, where renormalization is insignificant. With increasing excitation fluence $P$, we observe a threshold at $P_{\mathrm{thr}}=26 \mu \mathrm{J} / \mathrm{cm}^{2}$ at which the emission shrinks in momentum space as shown by the intensity profiles in Figs. 2(a)-2(c). Also above threshold, the emission at high $k$ is following the expected LP dispersion, confirming the strong coupling regime. The LP spectrum at $k \sim 0$ also displays the expected features for polariton condensation, namely, a linewidth narrowing in Fig. 2(d), a blueshift of the polariton mode in Fig. 2(e), and a nonlinear increase in intensity in Fig. 2(f). Increasing the fluence above threshold, interactions between the polariton condensate and the exciton reservoir increase, resulting in a broadening and blueshift of the condensate emission. ${ }^{25}$

To observe the transition from polariton condensation to photon lasing in the weak coupling regime, we need a

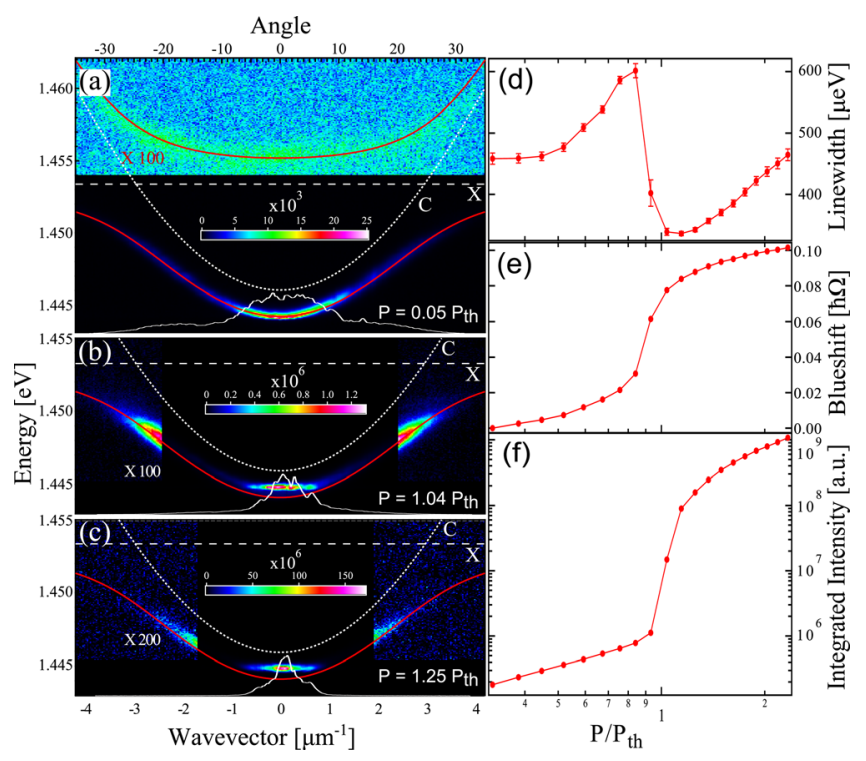

FIG. 2. Energy and wavevector resolved emission intensity on a linear color scale as indicated, (a) below, (b) at, and (c) above threshold. The red lines show the calculated LP and UP dispersions, and the dotted and dashed white lines show the uncoupled low-density cavity (C) and exciton (X) dispersion, respectively. In (a), the UP energy range is also shown, scaled as indicated. The data have been scaled in (b) for $|k|>2.5 \mu \mathrm{m}^{-1}$ and in (c) for $|k|>1.8 \mu \mathrm{m}^{-1}$ as indicated. Profiles of the LP emission along $k$ are also shown as white lines. (d) LP linewidth, (e) energy shift in units of the Rabi Splitting $2 \hbar \Omega$, and (f) intensity, at $k \sim 0$ versus excitation fluence. 
significantly higher excitation fluence, for which we reduce the excitation size to $9.2 \mu \mathrm{m}$ FWHM. The smaller excitation spot leads to polariton condensation at $|k|>0$ due to a steeper potential profile induced by the repulsive excitonexciton interactions in the reservoir. To record the evolution of the emission intensity with increasing excitation fluence between the two regimes, we integrate the emission over the entire LP, from $1.441 \mathrm{eV}$ to $1.458 \mathrm{eV}$ and $|k|<3.4 \mu \mathrm{m}^{-1}$. As shown in Fig. 3(a), we now find two thresholds, with the second one at about 20 times higher fluence than the first, showing an abrupt increase in intensity and decrease in linewidth. We note that directionally integrating the emission broadens the resulting linewidth compared to Fig. 2 and reduces the intensity difference between the linear and nonlinear plateau. Fig. 3(b) shows the energy shift of the emission color-coded with the average $\langle|\mathrm{k}|\rangle$ of the emission over the intensity distribution along the measured direction. As expected for a small excitation spot, the LP population build up occurs at $|k|>0$, increasing with the excitation fluence. However, upon crossing the second threshold, the emission shifts towards the energy of the uncoupled cavity mode and $k=0$. This second threshold is thus attributed to the transition to
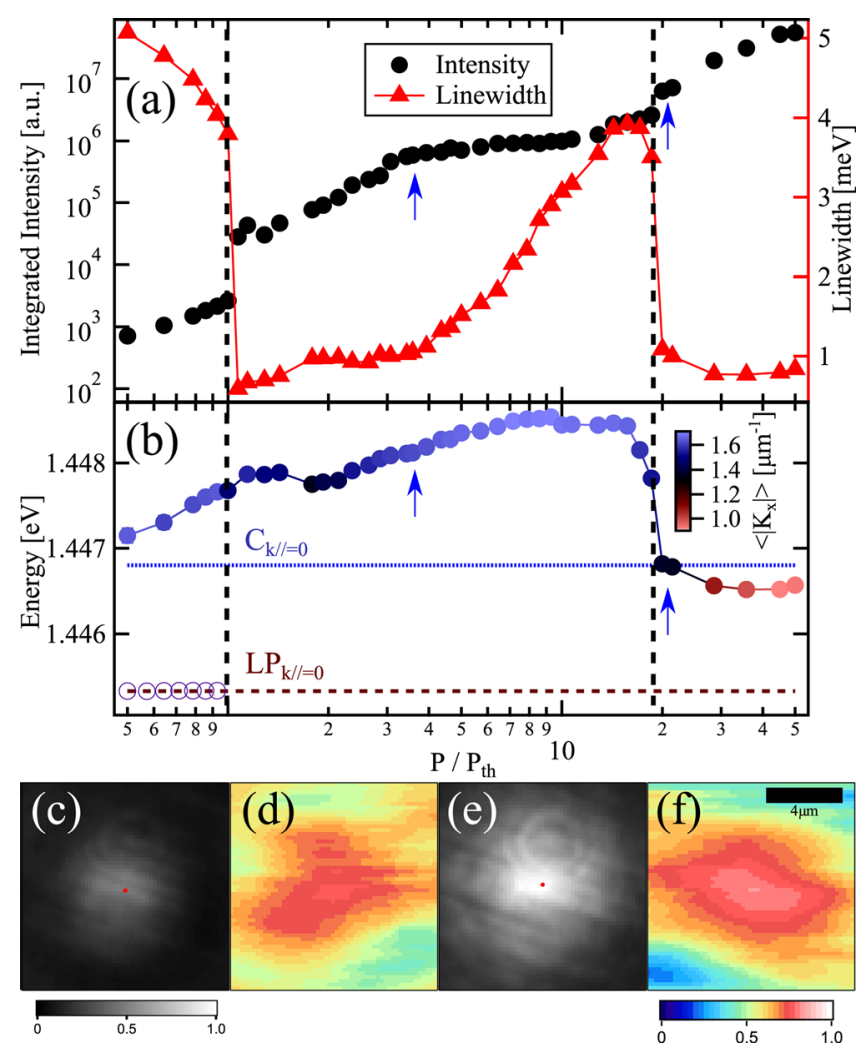

FIG. 3. LP emission using an excitation size of $9.2 \mu \mathrm{m}$ FWHM, as function of the excitation fluence $P$ relative to the polariton condensation threshold $P_{\text {th }}=28 \mu \mathrm{J} / \mathrm{cm}^{2}$. (a) Linewidth and spectrally integrated intensity, as a function of the excitation power. We integrate the emission over the entire LP, from $1.441 \mathrm{eV}$ to $1.458 \mathrm{eV}$ and $|k|<3.4 \mu \mathrm{m}^{-1}$. (b) Average energy, color coded with the average wavevector $\langle|k|\rangle$. The empty circles show the emission from the LP brunch at $k=0$ below threshold. The dotted lines indicate the energy of the cavity (blue) and the LP (brown) at $k=0$. (c) and (e) Spatially resolved emission intensity and (d) and (f) fringe visibility in the regime of polariton condensation at $P=3.8 P_{\text {th }}((\mathrm{c})$ and (d)) and photon lasing at $P=20 P_{\text {th }}((\mathrm{e})$ and (f)), as indicated by the two blue arrows in (a) and (b). The visibility is averaged over 24 different phase shifts. The red spots in (c) and (e) indicate $\mathbf{r}=0$ used in (d) and (f). photon lasing. ${ }^{26}$ To show the coherence build up above threshold, we carried out interference measurements using an actively stabilized Michelson interferometer in a mirrorretroreflector configuration ${ }^{3}$ measuring the coherence of the emission at $\mathbf{r}$ relative to $-\mathbf{r}$, with $\mathbf{r}=0$ set to the emission peak as indicated in Figs. 3(c) and 3(e). The extracted fringe visibility in the polariton condensate and photon lasing regime is shown in Figs. 3(d) and 3(f). The measured visibility $V$ of both the photon lasing and polariton condensate regime is extended and reaches up to about $80 \%$, consistent with the expected coherence of the emission. In conclusion, we have presented evidence of non-resonantly excited polariton condensation in a strain compensated GaAs-based cavity with InGaAs QWs. The observed nonlinear increase of intensity, along with a linewidth narrowing, and the observation of a second threshold to photon lasing identify this phase transition as polariton condensation in the strong coupling regime. As this type of strain compensated microcavity has been shown to suppress cross-hatched defects, ${ }^{19}$ it promises to be a suited system for studying the nature of quantum fluid phenomena. ${ }^{4,5,27}$

P.C., A.A., W.L., and P.G.L. acknowledge support by the EPSRC under Grant No. EP/F027958/1. The sample was grown by F.B. and E.C. at the EPSRC National Centre for III-V Technologies, Sheffield, UK.

${ }^{1}$ A. V. Kavokin, J. Baumberg, G. Malpuech, and F. P. Laussy, Microcavities (Oxford University Press, Oxford, 2007).

${ }^{2}$ A. Imamoglu, R. J. Ram, S. Pau, and Y. Yamamoto, "Nonequilibrium condensates and lasers without inversion: Exciton-polariton lasers," Phys. Rev. A 53, 4250-4253 (1996).

${ }^{3}$ J. Kasprzak, M. Richard, S. Kundermann, A. Baas, P. Jeambrun, J. M. J. Keeling, F. M. Marchetti, M. H. Szymanska, R. André, J. L. Staehli, V. Savona, P. B. Littlewood, B. Deveaud, and L. S. Dang, "Bose-Einstein condensation of exciton polaritons," Nature 443, 409-414 (2006).

${ }^{4}$ A. Amo, D. Sanvitto, F. P. Laussy, D. Ballarini, E. d. Valle, M. D. Martin, A. Lemaitre, J. Bloch, D. N. Krizhanovskii, M. S. Skolnick, C. Tejedor, and L. Viña, "Collective fluid dynamics of a polariton condensate in a semiconductor microcavity," Nature 457, 291-295 (2009).

${ }^{5}$ K. G. Lagoudakis, M. Wouters, M. Richard, A. Baas, I. Carusotto, R. André, L. S. Dang, and B. Deveaud-Plédran, "Quantized vortices in an exciton-polariton condensate," Nat. Phys. 4, 706-710 (2008).

${ }^{6}$ S. Christopoulos, G. B. H. von Högersthal, A. J. D. Grundy, P. G. Lagoudakis, A. V. Kavokin, J. J. Baumberg, G. Christmann, R. Butté, E. Feltin, J.-F. Carlin, and N. Grandjean, "Room-temperature polariton lasing in semiconductor microcavities," Phys. Rev. Lett. 98, 126405 (2007).

${ }^{7}$ F. Li, L. Orosz, O. Kamoun, S. Bouchoule, C. Brimont, P. Disseix, T. Guillet, X. Lafosse, M. Leroux, J. Leymarie, M. Mexis, M. Mihailovic, G. Patriarche, F. Réveret, D. Solnyshkov, J. Zuniga-Perez, and G. Malpuech, "From excitonic to photonic polariton condensate in a ZnO-based microcavity,” Phys. Rev. Lett. 110, 196406 (2013).

${ }^{8}$ K. S. Daskalakis, S. A. Maier, R. Murray, and S. Kéna-Cohen, "Nonlinear interactions in an organic polariton condensate," Nat. Mater. 13, 271-278 (2014).

${ }^{9}$ J. D. Plumhof, T. Stöferle, L. Mai, U. Scherf, and R. F. Mahrt, "Roomtemperature Bose-Einstein condensation of cavity exciton-polaritons in a polymer," Nat. Mater. 13, 247-252 (2014).

${ }^{10}$ E. Wertz, L. Ferrier, D. D. Solnyshkov, P. Senellart, D. Bajoni, A. Miard, A. Lemaitre, G. Malpuech, and J. Bloch, "Spontaneous formation of a polariton condensate in a planar GaAs microcavity,” Appl. Phys. Lett. 95, 051108 (2009).

${ }^{11}$ C. Schneider, A. Rahimi-Iman, N. Y. Kim, J. Fischer, I. G. Savenko, M. Amthor, M. Lermer, A. Wolf, L. Worschech, V. D. Kulakovskii, I. A. Shelykh, M. Kamp, S. Reitzenstein, A. Forchel, Y. Yamamoto, and S. Höfling, "An electrically pumped polariton laser," Nature 497, 348-352 (2013). 
${ }^{12}$ P. Bhattacharya, B. Xiao, A. Das, S. Bhowmick, and J. Heo, "Solid state electrically injected exciton-polariton laser,” Phys. Rev. Lett. 110, 206403 (2013).

${ }^{13}$ P. Bhattacharya, T. Frost, S. Deshpande, M. Z. Baten, A. Hazari, and A. Das, "Room temperature electrically injected polariton laser," Phys. Rev. Lett. 112, 236802 (2014).

${ }^{14}$ J. M. Zajac, W. Langbein, M. Hugues, and M. Hopkinson, "Polariton states bound to defects in gaas/alas planar microcavities," Phys. Rev. B 85, 165309 (2012).

${ }^{15}$ M. Gurioli, F. Bogani, D. S. Wiersma, P. Roussignol, G. Cassabois, G. Khitrova, and H. Gibbs, "Experimental study of disorder in a semiconductor microcavity,” Phys. Rev. B 64, 165309 (2001).

${ }^{16} \mathrm{~W}$. Langbein and J. M. Hvam, "Elastic scattering dynamics of cavity polaritons: Evidence for time-energy uncertainty and polariton localization," Phys. Rev. Lett. 88, 047401 (2002).

${ }^{17}$ W. Langbein, "Energy and momentum broadening of planar microcavity polaritons measured by resonant light scattering," J. Phys.: Condens. Matter 16, S3645 (2004).

${ }^{18}$ M. Abbarchi, C. Diederichs, L. Largeau, V. Ardizzone, O. Mauguin, T. Lecomte, A. Lemaitre, J. Bloch, P. Roussignol, and J. Tignon, "Discretized disorder in planar semiconductor microcavities: Mosaicity effect on resonant Rayleigh scattering and optical parametric oscillation," Phys. Rev. B 85, 045316 (2012).

${ }^{19}$ J. M. Zajac, E. Clarke, and W. Langbein, "Suppression of cross-hatched polariton disorder in GaAs/AlAs microcavities by strain compensation," Appl. Phys. Lett. 101, 041114 (2012).

${ }^{20}$ R. Houdré, J. L. Gibernon, P. Pellandini, R. P. Stanley, U. Oesterle, C. Weisbuch, J. O'Gorman, B. Roycroft, and M. Ilegems, "Saturation of the strong-coupling regime in a semiconductor microcavity: Free-carrier bleaching of cavity polaritons," Phys. Rev. B 52, 7810-7813 (1995).

${ }^{21}$ V. Savona, L. Andreani, P. Schwendimann, and A. Quattropani, "Quantum well excitons in semiconductor microcavities: Unified treatment of weak and strong coupling regimes," Solid State Commun. 93, 733-739 (1995).

${ }^{22}$ The transfer Matrix simulations were performed using the low temperature $(\sim 6 \mathrm{~K})$ refractive indices of 3.556 and 3.015 for the $\mathrm{GaAs}$ and $\mathrm{AlAs}_{0.98} \mathrm{P}_{0.02}$ layers, respectively, at the cavity resonance.

${ }^{23}$ D. Sanvitto, S. Pigeon, A. Amo, D. Ballarini, M. D. Giorgi, I. Carusotto, R. Hivet, F. Pisanello, V. G. Sala, P. S. S. Guimaraes, R. Houdré, E. Giacobino, C. Ciuti, A. Bramati, and G. Gigli, "All-optical control of the quantum flow of a polariton condensate," Nat. Photonics 5, 610-614 (2011).

${ }^{24}$ G. Nardin, G. Grosso, Y. Leger, B. Pietka, F. Morier-Genoud, and B. Deveaud-Pledran, "Hydrodynamic nucleation of quantized vortex pairs in a polariton quantum fluid," Nat. Phys. 7, 635-641 (2011).

${ }^{25}$ A. Askitopoulos, H. Ohadi, A. V. Kavokin, Z. Hatzopoulos, P. G. Savvidis, and P. G. Lagoudakis, "Polariton condensation in an optically induced two-dimensional potential," Phys. Rev. B 88, 041308 (2013).

${ }^{26}$ E. Kammann, H. Ohadi, M. Maragkou, A. V. Kavokin, and P. G. Lagoudakis, "Crossover from photon to exciton-polariton lasing," New J. Phys. 14, 105003 (2012).

${ }^{27}$ P. Cilibrizzi, H. Ohadi, T. Ostatnicky, A. Askitopoulos, W. Langbein, and P. Lagoudakis, "Linear wave dynamics explains observations attributed to dark solitons in a polariton quantum fluid," Phys. Rev. Lett. 113, 103901 (2014). 